\title{
Gene Shift Operators of Partheno-Genetic Algorithm for Permutation Flowshop Scheduling with Limited Waiting Times
}

\author{
Bailin Wang ${ }^{1,2}$, Haifeng Wang ${ }^{1,2}$, Tieke Li ${ }^{1,2}$ \\ ${ }^{1}$ Donlinks School of Economics and Management \\ University of Science and Technology Beijing \\ Beijing, China \\ ${ }^{2}$ Engineering Research Center of MES Technology for Iron \& Steel Production \\ Ministry of Education \\ Beijing, China \\ E-mail: wangbl@ustb.edu.cn
}

\begin{abstract}
Permutation flowshop scheduling with limited waiting times restricts maximum values of waiting times of jobs between consecutive machines. The problem with the objective of makespan is a NP-hard combination optimization problem. Considering characters of this scheduling problem, a basic Partheno-Genetic Algorithm (PGA) is presented, and constructions of two kinds of gene shift operators in PGA are focused on, including single-point shift operator and multi-point shift operator. Computational Experiments are carried out to evaluate the optimization performances of the two operators, and results show that single-point shift operator performs better in the basic PGA for this scheduling problem.
\end{abstract}

Keywords—permutation flowshop scheduling; partheno-genetic algorithm; limited waiting times; gene exchange operator

\section{INTRODUCTION}

Permutation flowshop scheduling problem with limited waiting times restricts the maximum values of waiting times of jobs between consecutive machines. The special waiting time constraints, which are called maximum time lag constraints as well, usually exist in a high degree of continuous production environment due to the instable physical or chemical nature of intermediate product, such as steel production [1], food production [2] and semiconductor manufacture [3].

This problem was firstly proposed by Yang and Chen in 1995 [4]. Fondrevelle, Oulamara and Portman [5] proved that this problem with makespan objective is a strong NP-hard combination optimization problem. For solving the problem, some algorithms were presented, including branch and bound method [4], mathematical programming [6] and heuristic algorithms $[7,8]$.

Partheno-Genetic Algorithm (PGA) is an improved genetic algorithm presented by $\mathrm{Li}$ and Tong [9] for combination optimization problems. In PGA, gene recombination operators are used to replace traditional crossover operators so that a new offspring can be generated by only one parent. In this paper, a basic process of PGA for the permutation flowshop scheduling problem with limited waiting times to minimize the makespan is discussed, and two kinds of gene shift operators in the basic PGA, which are single-point shift operator and multi-point shift operator, are designed with the consideration of problem characters. Comparative analysis of the two operators is made based on computational experiments.

\section{PROBLEM DESCRIPTION}

Permutation flowshop scheduling with limited waiting times to minimize the makespan considered in this paper can be formulated as follows.

There are $n$ jobs $\left\{J_{1}, J_{2}, \ldots, J_{i}, \ldots, J_{n}\right\}$ and $m$ machines $\left\{M_{1}, M_{2}, \ldots, M_{j}, \ldots, M_{m}\right\}$ in the workshop, where $i$ and $j$ represent job number and machine number respectively.

All the jobs must be processed by the same route as $M_{1} \rightarrow M_{2} \rightarrow \cdots \rightarrow M_{m}$. Denoting the processing time and completion time of $J_{i}$ on $M_{j}$ by $p_{i j}$ and $C_{i j}$, arriving constraints $C_{i 1} \geq p_{i 1}$ and temporal constraints $C_{i j}-C_{i, j-1} \geq p_{i j}$ must be satisfied, where $1 \leq i \leq n$ and $2 \leq j \leq m$.

Job sequence on each machine for processing is the same as others. Denoting the job sequence by $\pi$, and the $i$-th processed job by $\pi(i)$, there exist machine resource constraints that $C_{\pi(i), j}-C_{\pi(i-1), j} \geq p_{\pi(i), j}$, where $2 \leq i \leq n$ and $1 \leq j \leq m$.

The maximum values of waiting times are restricted. Denoting the waiting time and its allowed maximum value of $J_{i}$ between $M_{j}$ and $M_{j+1}$ by $w_{i j}$ and $\alpha_{i j}$, the limited waiting time constraints can be represented as $w_{i j}=C_{i, j+1}-C_{i j}-p_{i, j+1} \leq \alpha_{i j} \quad, \quad$ where $\quad 1 \leq i \leq n \quad$ and $1 \leq j \leq m-1$. 
The problem is to find an optimal job sequence $\pi^{*}$ for processing and determine the best completion times $\left\{C_{\pi(i), j} \mid 1 \leq i \leq n, 1 \leq j \leq m\right\}$ under this sequence to minimize the maximum completion time, which is equivalent to the makespan, represented as $\min C_{\max }=\max _{i, j}\left\{C_{i j}\right\}$.

The considered scheduling problem can be represented as $\mathrm{Fm} \mid$ prmu, $w_{i j} \leq \alpha_{i j} \mid C_{\max }$, and its solution space is $n$ !.

\section{PGA AND ITS GENE SHIFT OPERATORS}

\section{A. Basic Procedure of PGA}

In PGA, gene recombination operator and gene mutation operator are proposed as parthenogenesis to generate offspring. The algorithm support serial number codes so that it can ensure valid spring generation for combination optimization problems.

For the permutation flowshop scheduling problem, job numbers can be taken as gene codes to form a chromosome in PGA. Each chromosome represents a job sequence with the length $n$, and genes in a chromosome are all different. It is obviously that applying gene mutation operators will make the job sequence infeasible, thus only gene recombinant operators are adopted in this PGA, named basic PGA.

The flow chart of the basic PGA for Fm $\mid$ prmu, $w_{i j} \leq \alpha_{i j} \mid C_{\max }$ is shown in Fig. 1.

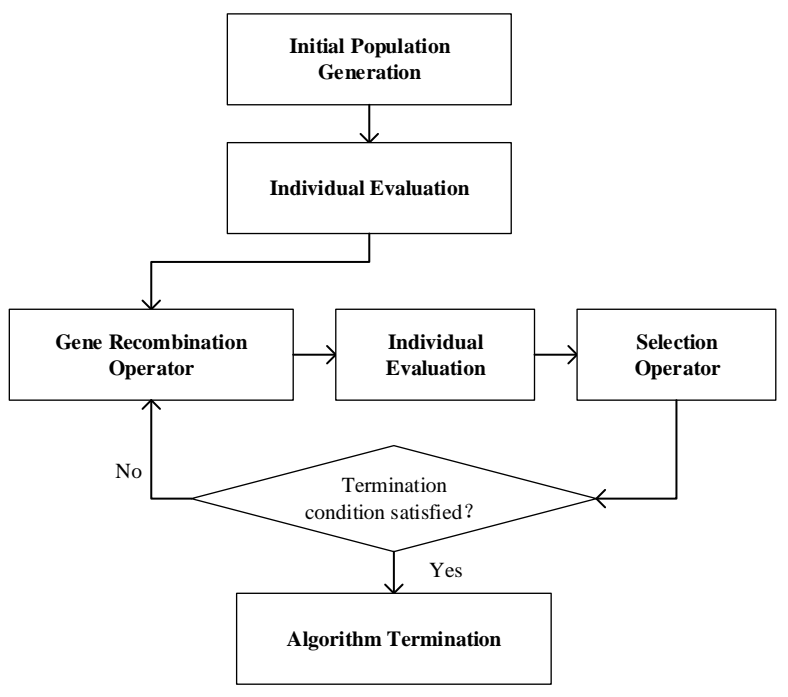

Fig. 1. Flow chart of the basic PGA for $\mathrm{F} m\left|p r m u, w_{i j} \leq \alpha_{i j}\right| C_{\max }$

In the basic PGA, the population size is equal to the length of a chromosome which is equal to $n$; initial population is generated by an extended NEH named ENEH with the consideration of limited waiting times. In ENEH, completion times under a partial schedule $\pi$ are calculated by the following procedure.

Step1 $C_{\pi(1), 1}=p_{\pi(1), 1} ;$ For $j=2$ to $m$, calculate (1);

$$
C_{\pi(1), j}=C_{\pi(1), j-1}+p_{\pi(1) j}
$$

Step2 For $i=2$ to $n$ do

(a) $C_{\pi(i), 1}=C_{\pi(i-1), 1}+p_{\pi(i) 1}$;

(b) For $j=2$ to $m$, calculate (2);

$$
C_{\pi(i), j}=\max \left\{C_{\pi(i-1), j}, C_{\pi(i), j-1}\right\}+p_{\pi(i), j}
$$

(c) For $j=m-1$ to 1 , calculate (3);

$$
C_{\pi(i), j}=\max \left\{C_{\pi(i), j}, C_{\pi(i), j+1}-p_{\pi(i), j+1}-\alpha_{i j}\right\}
$$

Step3 $C_{\max }(\pi)=C_{\pi(n), m}$.

Moreover, in this basic PGA, the individual fitness function is defined as $C_{\max }=\max _{i, j}\left\{C_{i j}\right\}$, and the selection operator applies tournament method to choose the top $n$ individuals for the fitness of makespan from all the parents and their children to form a new population. The termination condition is set as the maximum number of iteration $t$ : If the iteration number is larger than $t$, then the algorithm is stopped and the best individual and its makespan is output.

\section{B. Gene shift operators}

Gene recombination operators can be divided into three classes, including gene shift operators, gene exchange operators and gene inverse operators. The constructions of gene shift operators will be focused on in this section.

Gene shift operators refer to a shifting operation to choose one or more genes and the same number of positions in one chromosome, and insert the genes into the given positions to form a new individual. Considering the characters of the problem $\mathrm{Fm} \mid$ prmu, $w_{i j} \leq \alpha_{i j} \mid C_{\max }$, two kinds of gene shift operators are presented below.

\section{1) Single-Point Shift Operator}

Single-point shift operator is to select only one gene and one position during one shift operation. It should be noticed that the selected position must be different from the current position of the selected gene.

For example, a chromosome is $\{532614\}$, which means that the job sequence for processing is $J_{5}$ first, $J_{3}$ second, then $J_{2}, J_{6}$ and $J_{1}$, and $J_{4}$ at last. A single-point shift operator is carried out with the gene " 6 " and the position " 2 ", thus a new chromosome is generated as $\{563214\}$, where $J_{6}$ is the second processed job, and $J_{3}$ and $J_{2}$ are postponed after $J_{6}$.

It is clear that the initial position of $J_{6}$ in the parent chromosome is 4 , and if the selected position is 4 as well, the 
child is just the same as its parent, which is means that the gene shift operator makes no sense. Therefore, the following rules are suggested for single-point shift operator.

Rule 1. The gene should be first selected, and then the position.

Rule 2. The selected position cannot be equal to the original position of the selected gene.

\section{2) Multi-point Shift Operator}

In this multi-point shift operator, a maximal shift value is specified first, and then pairs of genes and positions are selected randomly with the number that is no larger than the maximal shift value, finally the shift operations are carried out in order. Actually, this shift operator should be more accurately called as random multi-point shift operator because the number of shift points is not fixed in each shift operation.

For the problem $\mathrm{Fm} \mid$ prmu, $w_{i j} \leq \alpha_{i j} \mid C_{\max }$, the following rules are put forward for multi-point shift operator.

Rule 3. The maximum shift value is set as $n$.

Rule 4. To increase the population diversity, the selected jobs should be different from others.

Taken the above example $\{532614\}$ as well, the maximum shift value is 6 , which means that the number of selected pairs of genes and positions should belong to $[1,6]$. Now a twopoint shift operator is carried out with two pairs of genes and their new positions as $(1,3)(2,5)$, where the first element in one pair of parentheses is the selected gene, and the second represents the position. The first shift operation is to insert $J_{1}$ into Position 3, and $\{531264\}$ is obtained; after the second shift operation of $(2,5)$, a new individual is generated as $\{531624\}$.

It can be seen that the multi-point shift operator may make greater changes than the single-point shift operator. So comes the question whether great changing of a parent chromosome is good for solving the considered scheduling problem or not? In the next section, computational experiences are carried out to compare the performances of the basic PGAs with the two gene shift operators.

\section{COMPUTATIONAL EXPERIMENTS}

\section{A. Experiment Design}

In this section, optimization performances of the two shift operators are compared by computational experiments. For ease of description, algorithms with the two operators are respectively named as PGAS (short for Basic PGA with Single-Point Shift Operator) and PGAM (short for Basic PGA with Multi-Point Shift Operator). The two algorithms are coded in C\# language and implemented on the computer with Intel Core i3-4020Y/ CPU1.5GHz/ RAM4.0G.

In the experiments, the parameters of test problems are set as $n=\{20,40,60,80,100\}, m=\{4,6,8,10\}, p_{i j} \in D U[1,99]$, and $\alpha_{i j} \in D U[1,99]$. According to the different problem sizes, the experiments are divided into 20 groups, and each group generated 10 test problems randomly. The maximum iteration numbers of the two algorithms are both set as 200 .

Performance measurements considered here mainly focus on the effectiveness and efficiency of algorithms. For the former, the improving ratio on the makespan of the initial solution obtained by ENEH is considered, denoted by $I R$. Its computing formula is in (4).

$$
I R=\frac{C_{\max }^{0}-C_{\max }^{*}}{C_{\max }^{0}} \times 100 \%
$$

In (4), $C_{\max }^{*}$ is the best makespan obtained by the algorithm, and $C_{\max }^{0}$ is the makespan of the initial solution. For the latter, the measurement of computing time is adopted, denoted by $T$.

\section{B. Experimental Results}

Computing results are shown in Table 1.

TABLE I. COMPUTING RESULTS

\begin{tabular}{|c|c|c|c|c|}
\hline \multirow{2}{*}{$n \times m$} & \multicolumn{2}{|c|}{ PGAS } & \multicolumn{2}{|c|}{ PGAM } \\
\hline & $\overline{I R} / \%$ & $\bar{T} / \mathbf{s}$ & $\overline{I R} / \%$ & $\bar{T} / \mathbf{s}$ \\
\hline $20 \times 4$ & 1.973 & 0.056 & 0.771 & 0.078 \\
\hline $20 \times 6$ & 1.413 & 0.081 & 0.760 & 0.104 \\
\hline $20 \times 8$ & 2.479 & 0.107 & 0.817 & 0.131 \\
\hline $20 \times 10$ & 2.201 & 0.131 & 0.890 & 0.155 \\
\hline $40 \times 4$ & 2.042 & 0.252 & 0.517 & 0.344 \\
\hline $40 \times 6$ & 1.884 & 0.406 & 0.258 & 0.512 \\
\hline $40 \times 8$ & 2.153 & 0.513 & 0.595 & 0.611 \\
\hline $40 \times 10$ & 1.954 & 0.645 & 0.444 & 0.744 \\
\hline $60 \times 4$ & 0.852 & 0.638 & 0.116 & 0.870 \\
\hline $60 \times 6$ & 0.964 & 1.020 & 0.158 & 1.260 \\
\hline $60 \times 8$ & 1.672 & 1.345 & 0.234 & 1.573 \\
\hline $60 \times 10$ & 1.423 & 1.725 & 0.191 & 1.972 \\
\hline $80 \times 4$ & 0.619 & 1.278 & 0.027 & 1.684 \\
\hline $80 \times 6$ & 0.921 & 2.085 & 0.165 & 2.485 \\
\hline $80 \times 8$ & 0.618 & 2.774 & 0.065 & 3.201 \\
\hline $80 \times 10$ & 0.807 & 3.520 & 0.115 & 4.007 \\
\hline $100 \times 4$ & 0.390 & 2.236 & 0.066 & 2.891 \\
\hline $100 \times 6$ & 0.542 & 3.602 & 0.092 & 4.340 \\
\hline $100 \times 8$ & 0.474 & 4.923 & 0.042 & 5.561 \\
\hline $100 \times 10$ & 0.521 & 6.244 & 0.074 & 7.019 \\
\hline
\end{tabular}

From Table 1, it can be seen that for the performances of effectiveness, single-point shift operator was evidently superior to multi-point shift operators, although the latter may make greater changes from parent chromosomes. Moreover, in 
the 200 randomly generated instances, the best improving ratio of the basic PGA with single- and multi-point shift operator are $6.429 \%$ and $3.972 \%$ respectively. It also indicates that single-point exchange operator has better performance for the considered scheduling problem.

Concerning the performance of efficiency, computing times of the both two algorithms are very fast. Even for the problem with 100 jobs and 10 machines, the two algorithms could obtain schedules in $8 \mathrm{~s}$.

To further evaluate the influence of these operators on the performance of PGA, convergences of the two algorithms for an instance with 20 jobs and 6 machines are given in Figure 2. The improving ratios of PGAS and PGAM for this instant are $6.241 \%$ and $2.482 \%$ respectively.

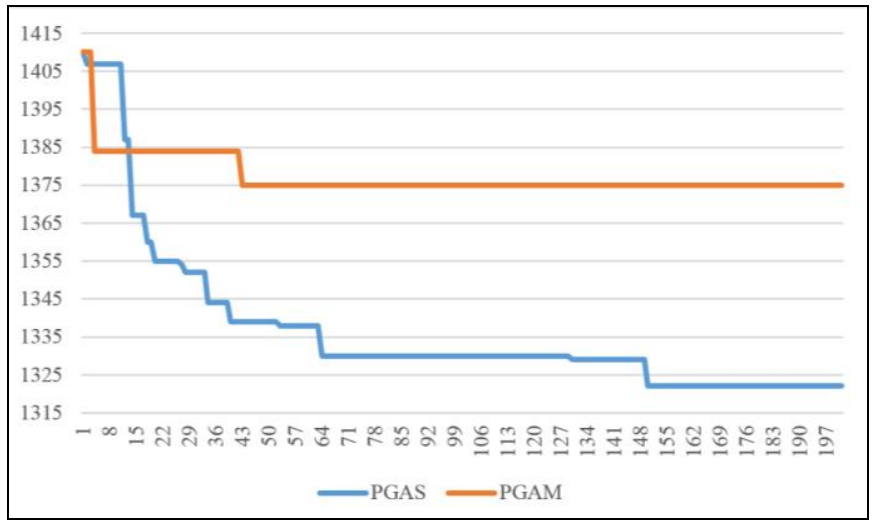

Fig. 2. Convergences of the two algorithms

As Fig. 2 shown, these PGAs are all convergent before the 160th iteration, and PGAS which adopts single-point shift operator has better advantages in solution search and continual optimization.

\section{CONCLUSIONS}

Permutation flowshop scheduling problem with limited waiting times is a NP-hard combination optimization problem with special constraints that waiting times of jobs between any two consecutive machines cannot be larger than the given maximum values. For the problem to minimize the makespan, a basic partheno-genetic algorithm is presented, in which the initialization population generation method, fitness function, selection operator, and termination condition are discussed. For further optimization, two kinds of gene shift operators, single-point shift operator and multi-point shift operator, are focused on. The constructions of these two operators are put forward with characteristics of the considered scheduling problem, and their optimization performances are compared by computational experiments. Experimental results show that single-point shift operator has better effectiveness in the basic PGA for this scheduling problem, and both the PGAs with the two operators have good solving efficiency.

\section{ACKNOWLEDGMENT}

This work is financially supported by China Scholarship Council, Fundamental Research Funds for the Central Universities (No. FRF-BR-14-011A), and China Postdoctoral Science Foundation (No. 2012M510324).

\section{REFERENCES}

[1] K. Mao, Q-K. Pan, X. Pang, and T. Chai, "A novel Lagrangian relaxation approach for a hybrid flowshop scheduling problem in the steelmaking-continuous casting process," European Journal of Operational Research, vol. 236, pp. 51-60, July 2014.

[2] T. Wauters, K. Verbeeck, P. Verstraete, G. V. Berghea, and P. D. Causmaecker, "Real-world production scheduling for the food industry: An integrated approach," Engineering Applications of Artificial Intelligence, vol. 25, pp. 222-228, March 2012.

[3] H-K. Wang, C-F. Chien, and M. Gen, "Hybrid estimation of distribution algorithm with multiple subpopulations for semiconductor manufacturing scheduling problem with limited waiting-time constraint," 2014 IEEE International Conference on Automation Science and Engineering (CASE), Taipei, Taiwan, 2014.

[4] D.L. Yang and M.S. Chern, "A two-machine flowshop sequencing problem with limited waiting time constraints," Computers and Industrial Engineering, vol. 28, pp. 63-70, Jan. 1995.

[5] J. Fondrevelle, A. Oulamara and M. C. Portmann, "Permutation flowshop scheduling problems with maximal and minimal time lags," Computers \& Operations Research, vol. 33, pp. 1540-1556, June 2006.

[6] E. Dhouib, J. Teghem, and T. Loukil, "Minimizing the Number of Tardy Jobs in a Permutation Flowshop Scheduling Problem with Setup Times and Time Lags Constraints," Journal of Mathematical Modelling and Algorithms in Operations Research, vol 12, pp. 85-99, March 2013.

[7] L.H. Su, "A hybrid two-stage flowshop with limited waiting time constraints," Computers \& Industrial Engineering, vol 44, pp. 409-424, March 2003.

[8] B. Wang, T. Li, C. Shi, and H. Wang, "Scheduling two-machine flowshop with limited waiting times to minimize makespan," TELKOMNIKA Indonesian Journal of Electrical Engineering, vol 12, pp. 3131-3139, April 2014.

[9] M. J. Li and T. S. Tong, "A partheno genetic algorithm and analysis on its global convergence" Acta Automat Sin, vol 25, Jan. 1999, pp. 68-72. (in Chinese) 